\title{
LIX. On measurements of the intensity of the horizontal component of the earth's magnetic field made in the physical laboratory of the University of Glasgow
}

Thomas Gray B.Sc. F.R.S.E.

To cite this article: Thomas Gray B.Sc. F.R.S.E. (1885) LIX. On measurements of the intensity of the horizontal component of the earth's magnetic field made in the physical laboratory of the University of Glasgow, Philosophical Magazine Series 5, 20:127, 484-497, DOI: 10.1080/14786448508627793

To link to this article: http://dx.doi.org/10.1080/14786448508627793

曲 Published online: 29 Apr 2009.

Submit your article to this journal $₫$

Џll Article views: 2

Q View related articles ¿ 


\section{[ 484$]$}

LIX. On Measurements of the Intensity of the Horizontal Component of the Earth's Magnetic Field made in the Physical Laboratory of the Uninersity of Glasgow. By THоmas GraY, B.Sc., F.R.S.E.*

[Plate XI.]

T $N$ the course of some measurements of the horizontal component of the intensity of the earth's magnetic field, which have recently been made in the physical laboratory of the University of Glasgow, several improvements in the apparatus and mode of conducting these experiments suggested themselves. The following paper is a description of these measurements.

The method adopted was, in general principle, that of Gauss ; that is, the determination by a deflection-experiment of the ratio $M / H$, and by an oscillation-experiment of the product $\mathrm{MH}$, where $\mathrm{M}$ is the magnetic moment of a magnet, called in this description the deflector, and $\mathrm{H}$ is the horizontal intensity of the earth's magnetic field at the place of experiment. The earlier experiments, of which the results are given below, were, with the exception of the mode of determining the effective length of the deflector, conducted in the way that has for several years been practised in Sir William Thomson's laboratory, and descriptions of which have already been publishedt.

The Deflection Experiment consists in finding the deflection produced by the deflector on a second needle, called the magnetometer-needle, suspended at a known distance from it, and thus furnishing an equation of the form

$$
\frac{\mathrm{M}}{f\left(2 a_{1}, r\right)}=\mathrm{H} \tan \theta ; . . . \quad . \quad . \quad .
$$

where $f\left(2 a_{1}, r\right)$ is a function of $2 a_{1}$ the effective length of the deflector, and $r$ the distance between the centre of the deflector and the centre of the magnetometer-needle, and $\theta$ is the angle of deflection. The function $f\left(2 a_{1}, r\right)$ depends on the relative direction of the line joining the centres of the deflector and magnetometer-needles, the direction of the axis of the defiector and the direction of the lines of force in the magnetic field, and on the distribution of magnetism in the deflector and magnetometer-needles. Strictly $f\left(2 a_{1}, r\right)$ should be $f\left(2 a_{1}, b, r\right)$,

* Paper read before the British Association at the Aberdeen Meeting, September 1885. Communicated by Sir William Thomson, F.R.S.

+ Vide Phil. Mag. for November 1878; 'Electrician' for July 8, 1882

'Nature' for November 9, 1882. 
where $b$ is the length of the magnetometer-needle; but it is one of the merits of the method here described that the magnetometer-needle is made so short that its magnetic length may be neglected, and the resulting equation thus much simplified.

The positions adopted in these determinations were, for the line joining the centres of the deflector and the magnetometerneedle, the magnetic meridian and a line at right angles to it ; and for the direction of the magnetic axis of the deflector, a line at right angles to the magnetic meridian. We then have for the former of these positions,

$$
f\left(2 a_{1}, r\right)=\frac{\left(r^{2}-a_{1}^{2}\right)^{2}}{2 r} ; . . . . .
$$

and for the latter position,

$$
f\left(2 a_{1}, r\right)=\left(r_{1}^{2}+a_{1}^{2}\right)^{\frac{3}{2}} \text {. . . . . . . }
$$

From equation (1) we obtain, by (2) and (3),

and

$$
\overline{\mathrm{M}}=\frac{\left(r^{2}-a_{1}^{2}\right)^{2}}{2 r} \tan \theta_{0}, \ldots . . . \text {. . }
$$

$$
\overline{\mathrm{M}}=\left(r_{1}^{2}+a_{1}^{2}\right)^{\frac{3}{9}} \tan \theta_{1} . \quad \text {. . . . }
$$

Now, besides $M$ and $H$, the value of $a_{1}$ is also unknown ; but when $\theta_{0}$ and $\theta_{1}$ are determined at nearly the same time, we can calculate $a_{1}$ from the above equations. We have clearly

$$
\frac{\left(r^{2}-a_{1}^{2}\right)^{2}}{2 r\left(r_{1}^{2}+a_{1}^{2}\right)^{\frac{3}{2}}}=\frac{\tan \theta_{1}}{\tan \theta_{0}} \text {. . . . . . }
$$

Expanding the numerator and denominator on the left-hand side of the above equation, and neglecting small terms, we readily obtain, as a close approximation,

$$
a_{1}^{2}=\frac{\theta_{0} r^{3}-2 \theta_{1} r_{1}^{3}}{2 \theta_{0} r^{2}+3 \theta_{1} r_{1}} . . . . . . .
$$

There is in the method here described a departure from the usual practice-namely, determining the effect of the length of the deflector and deflected magnets, by double and triple experiments with the deflector placed at different distances along a line through the centre of the deflected magnet. In the first place, the third experiment is rendered unnecesPhil. Mag. S. 5. Vol. 20. No. 127. Dec. 1885. 2 M 
sary by making the deflected magnet so short that its magnetic length may be neglected; and, in the second place, the effect of the length of the deflector is found by adouting two positions for which the length enters in the resulting equations (4) and (5) above, with opposite sign, rendering the observation highly sensitive to that effect. By adopting this method a good estimate of the effective length of different deflectors is obtained; and this is useful information outside of the particular object of the experiment.

The latest form and arrangement of the apparatus for the deflection-experiment is shown in $\mathrm{Pl}$. XI. fig. 1, where $T$ is the table on which the apparatus is placed, $M$ the magnetometer, $\mathrm{A}$ and $\mathrm{B}$ the deflector-stands, and $\mathrm{C}$ the scale on which the deflections are read. The magnetometer $M$ consists of a light mirror about $\cdot 8$ centim. in diameter, on the back of which two magnets, 1 centim. long and 08 centim. in diameter, are fixed. A better form of needle would, as has been pointed out by Sir William Thomson, be two thin disks of hard steel mounted with their planes parallel and at a distance apart somewhat less than the diameter of the disk.

The mirror with attached magnets is suspended by a single silk fibre (half a cocoon-fibre) in a recess cut in a block of wood, W. Two holes at right angles to each other, and passing through the position of the mirror and magnets, allow the magnetic system to be accurately adjusted when setting up the apparatus. The holes, with the exception of that in front of the mirror, are plugged while the instrument is in use, and the mirror and fibre are protected from currents of air by means of a plate of plane glass. The sole plate, $\mathrm{P}$, is furnished with three brass feet, which rest on a "hole, slot, and plane" arrangement cut in the top of the glass plate, $p$, fixed to the table. The deflector-stands, $A$ and $B$, consist of a base plate of mahogany furnished, as in the case of the magnetometer, with three brass feet which rest on "hole, slot, and plane" arrangements cut in the glass plate $p$. A centre pivot, $c$, is fixed in the sole plate and passes throngh a closely-fitting hole in the glass plate, $g$, which rests on three thin blocks of hard wood and is free to turn in azimuth. A strip of wood having a $\mathrm{V}$-groove cut along its upper side and furnished with an adjusting-screw, $s$, is cemented to the top of the glass plate $g$ in such a way that the bottom of the $V$-groove is vertically above the centre of the pivot $c$. The screw, $s$, gives an adjustment for the centre of the magnet, which is necessary if the magnets differ slightly in length. The centre of the magnet is seldom exactly at the middle of the length of the bar, but, with the arrangement here adopted, the adjustment 
Phil. Mag. S. 5. Vol . 20 . Pl. XI
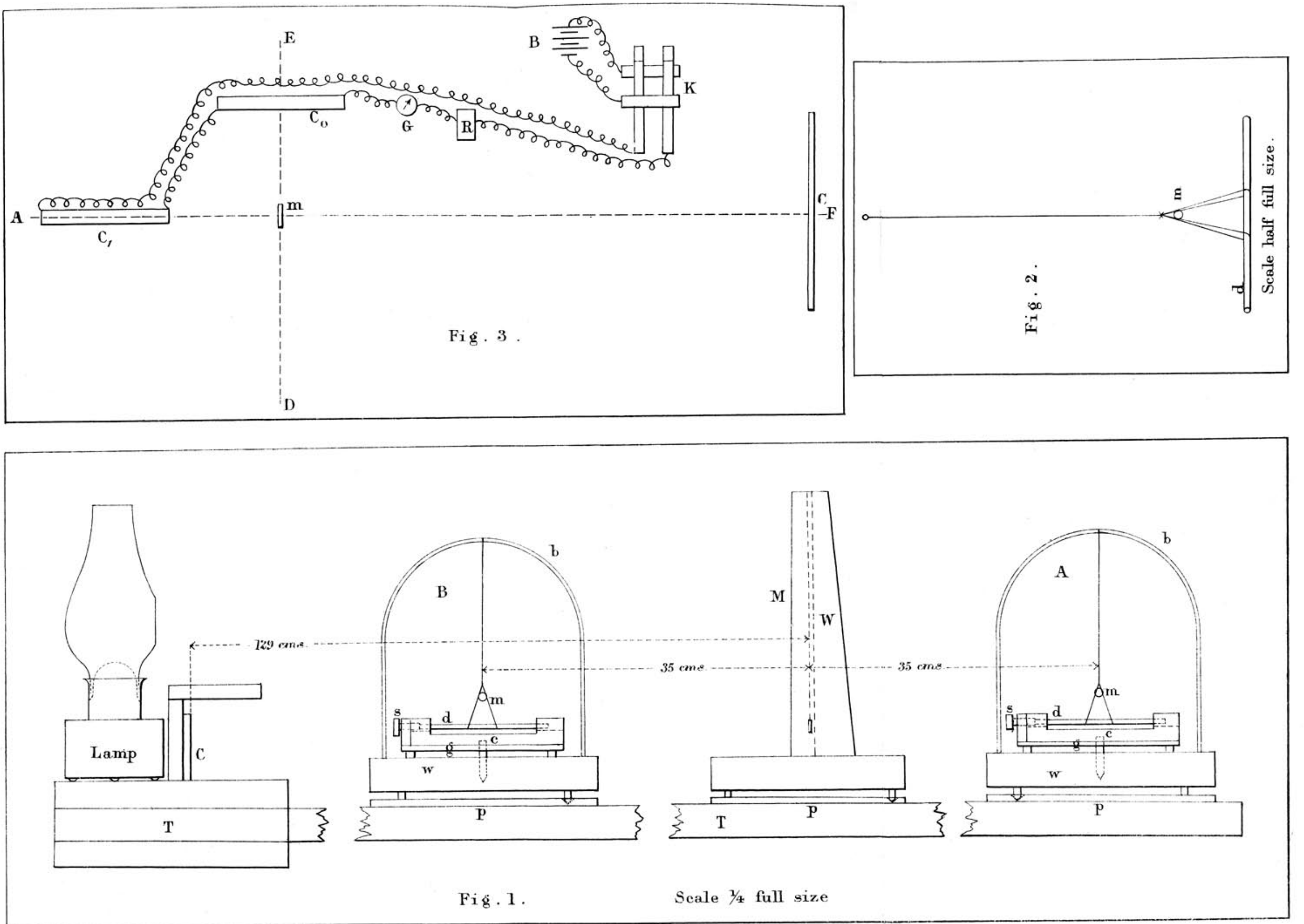
can be readily made by turning the screw $s$ until equal deflections are obtained on opposite sides of zero, when the deflector is reversed by turning the plate $g$ through $180^{\circ}$ in azimuth. The deflectors, $d$, are shown in positions east and west of the magnetometers; and the distance between their centres when in this position is 70 centim. Two plates similar to $p$ are placed, one on the north and one on the south side of the magnetometer, in such a position that, when the deflector-stand is resting on them, the distance between the centres of the deflectors is 60 centim., and the line joining them is in the magnetic meridian. The feet on which the deflector-stands rest are so adjusted that the deflectors are equidistant from the magnetometers, and at the same distance apart when the positions of the stands are interchanged. The scale $\mathrm{C}$ is graduated to millimetres on glass, and is placed with its centre directly in front, and in the focus, of the magnetometer-mirror. The distance between the scale and the mirror is 129 centim.

A paraffin-lamp, with a copper funnel which has a vertical slit in front, with a fine wire in its centre, covered by a plane glass plate, is placed behind the scale, and farnishes a beam of light which is focused on the glass scale by the magnetometermirror. The scale being transparent, the deflections can be read either from the back or the front of it. When the deflections were read from bebind the scale, it was found convenient, although not absolutely necessary, to render the glass partially obscure; and this, acting on a suggestion of Mr. Bottomley, was done by dusting lycopodium-powder over it. The deflections were taken in the following manner :-

The table $\mathrm{T}$ was first placed in such a position that the line joining the centres of $\mathrm{A}$ and $\mathrm{B}$ (Pl. XI. fig. 1) was exactly at right angles to the magnetic meridian. This was done in one or other of the following ways :- (1)A thin wire was passed under the magnetometer and stretched along the line joining the centres of $\mathrm{A}$ and $\mathrm{B}$, and then taken back, either over the top of the magnetometer, or beneath and at a greater distance from it, in such a way as to form a vertical plane circuit. An electric current was then sent through the circuit, and the table turned until it produced no deflection on the magnetometer-needle. (2) One of the deflectors was placed in its position north or south of the magnetometer, and lifted out of its $V$ by the suspension-fibre. The table was then turned until the suspended needle produced no deflection of the magnetometer-needle. When this is the case, the direction of the magnetic axes of the deflector and the magnetometer-needle are in the same line ; and if the latter needle be in its proper position, this line also passes through the centro of the deflector when placed on the $2 \mathrm{MI} 2$ 
other side of the magnetometer. To ensure that this was the case, the deflector was placed on the other side and the position of the table for no deflection again observed. The magnetometer-needle was then adjusted, by turning the levellingscrews, until the two positions were coincident. A combination of the methods (1) and (2) gives a ready means of testing whether the plates $g$ have been properly placed on the tables

Suppose A to be east and B west of the magnetometer. The deflectors were turned by means of the plate $g$ until their lengths were accurately in the magnetic east and west line, and their poles so placed as to produce a deflection to the same side of zero. The deflection was then read. The plates $g$ were next turned through $180^{\circ}$ and the deflection on the opposite side of zero read. The plates $g$ were then turned back to their first position, and the deflection again read. The difference between the mean of the first and third reading and the second gives twice the deflection. The same operation was then repeated with $A$ north and $B$ south, with $A$ west and $B$ east, and with $A$ sonth and $B$ west. The mean of the deflections for the east and west positions and the mean of the deflections for the north and south positions were then found, and from them the mean effective length of the two defectors calculated. This length was then substituted in equations (11) and (12) below, and the value of $\mathrm{H}$ calculated.

Previous to the adoption of the above apparatus only one deflector was used, and it was placed by hand in the positions necessary for a cycle of operations similar to that above described. The idea of using two deflectors placed on opposite sides of the magnetometer, and the arrangement for them shown in fig. 1, is due to Sir William Thomson. It has the advantage of greater symmetry, it allows the deflector to be placed at a greater distance from the magnetometer, and, what is most important, the magnet need not be handled during the experiment.

The Osclllation Experiment consists in finding the period of oscillation of the deflector when suspended with its axis horizontal, and free to perform horizontal oscillations under the influence of the earth's magnetic field. This experiment gives the value of $\mathrm{MH}$ from the equation

$$
\mathrm{MH}+x=\frac{4 \pi^{2} \mu}{\mathrm{P}^{2}} . \quad \text {. . . . . }
$$

The quantity $x$ refers to the torsional rigidity of the suspension, which was practically zero in these experiments ; $\mu$ is the moment of inertia of the oscillating system, and $\mathrm{P}$ the 
period of oscillation. The following is a description of the arrangement and the mode of observation.

A length of single cocoon-fibre was taken, and a stirrup like that shown at $\$$ (fig. 2) formed on one end of it by folding it twice, so as to make four fibres, and making a knot about 3 centim. from the end. The other end was passed through a small hole in the brass bow, $b$ (fig. 1), and then fixed to a small strip of sheet lead which simply rested on the sole plate and held the deflector in the proper position. The deflector was placed in the stirrup $S$, and the loops adjusted so as to suspend it horizontally. The magnet was thus suspended in a stirrup almost devoid of inertia, and by a fibre of negligible torsional rigidity. This leaves $\mu$, the moment of inertia of the deflector, and $P$, its period of oscillation, to be determined. The moment of inertia can be very accurately calculated from the mass, length, and thickness of the bar, when, as is the case with these deflectors, the bar is a round cylinder of small and perfectly uniform diameter. Greater accuracy in these calculations, and the advantage of small diameter in the "side on" position when taking deflections, are the main reasons, apart from simplicity, why a solid cylinder is preferred to a thin tube for the deflector. The period of oscillation $\mathrm{P}$ was in the earlier experiments obtained either by observing, with the eje placed behind a narrow slit, the times of successive transits of the end of the bar across a fixed mark (usually a black thread stretched vertically near the end of the magnet and in the same magnetic meridian); or by ob. serving the times of the successive transits across the vertical wire of a telescope. In the later experiments, one end of the deflector was polished with the view of using it as a mirror to reflect a beam of light to a scale placed at some distance. This method was not successful, owing to defects in the mirror, and it has been abandoned in favour of a light silvered-glass mirror, $m$ (fig. 1 ), about 0.3 centim. in diameter and 0.01 gramme in weight, attached to the stirrup with its plane parallel to the length of the magnet. The same lamp and scale are thus available both for oscillation and deflection experiments. With this arrangement the amplitude of the oscillation need never exceed one degree, and hence no correction for are is necessary.

With regard to the effect of the inertia of the mirror on $\mu$, it is to be remarked that its total moment of inertia is about .001 , while that of the deflector is about 40 ; so that, even if the inertia of the mirror be neglected, the error is not more than $\frac{1}{400}$ per cent.

When a second observer was available, time was usually 
taken from a watch with a centre seconds-hand moving over a dial divided to quarter-seconds. The watch was keeping almost perfect time. In this case an observer counted the oscillations of the magnet, and called now at the end of every four or five periods, while the other observer noted the time. Personal error does not enter into this method, as it is eliminated in taking the differences. When the transits and time were taken by the same observer, the time was taken from a chronometer beating half-seconds. The observer took time, say, at the beginning of a minute, and then counted the beats nntil he could observe a transit. By simply counting the number of beats between two successive transits, the total number of periods in one minute could then be estimated; and the time of the first transit after each minute was then taken as long as the amplitude was sufficiently large to allow the time to be accurately estimated. Fractions of half seconds were estimated from the position of the magnet at the beat next before and next after transit.

The results of the different observations of time were combined in the following manner:-First suppose an even number $2 n$ observations to have been taken. The sum of the intervals of time between the $n$th and the $(n+1)$ th, the $(n-1)$ th and the $(n+2)$ th, and so on to the 1 st and $2 n$ th, divided by the square of the number of intervals, and by the number of periods between each pair of observations, gives the average period as nearly as it can be obtained from that set of observations. Suppose next an odd number $2 n+1$ observations to have been taken. The sum of the intervals between the 1 st and the $(n+1)$ th, the 2 nd and $(n+2)$ th, and so on to the $n$th and $(2 n+1)$ th, each divided by the corresponding number of periods, divided by the number of intervals, gives the average period. Two sets of observations were usually taken; and if they agreed closely, the mean of the two was assumed to be the true period.

We have then the following equations for the determination of $\mathrm{H}$ when one deflector only is used :-

$$
\text { and } \quad \begin{aligned}
& \frac{\mathrm{M}}{\overline{\mathrm{H}}}=\frac{\left(r^{2}-a_{1}^{2}\right)^{2}}{2 r} \tan \theta_{0}, \quad . \quad . \quad . \quad . \\
& \overline{\mathrm{M}}=\left(r_{1}^{2}+a_{1}^{2}\right)^{\frac{3}{2}} \tan \theta_{1}, \quad . \quad . \quad . \quad . \\
& \mathrm{MH}=\frac{4 \pi^{2} \mu}{\mathrm{P}^{2}} \\
&=\frac{8}{3} \frac{\pi^{2}\left(a^{2}+\frac{3}{64} d^{2}\right) w}{\mathrm{P}^{2}}, \ldots . . .
\end{aligned}
$$


where $2 a$ is the length, $d$ the diameter, and $w$ the weight of the deflector-bar. From (4) and (8) we obtain,

From (5) and (8),

$$
\mathrm{H}^{2}=\frac{8}{3} \frac{\pi^{2}\left(a^{2}+\frac{3}{64} d^{2}\right) r w}{\mathrm{P}^{2}\left(r^{2}-a_{1}^{2}\right)^{2} \tan \theta_{0}} . \quad . \quad . \quad .
$$

$$
\mathrm{H}^{2}=\frac{4}{3} \frac{\pi^{2}\left(a^{2}+\frac{3}{32} d^{2}\right) w}{\mathrm{P}^{2}\left(r_{1}^{2}+a_{1}^{2}\right)^{\frac{3}{2}} \tan \theta_{1}} . . . .
$$

When two deflectors are used, as described above, equations (9) and (10) take the form,

$$
\mathrm{H}^{2}=\frac{8}{3} \frac{\pi^{2} r\left(a^{2}+\frac{3}{64} d^{2}\right)\left(\mathrm{P}_{1}^{2} w_{2}+\mathrm{P}_{2}^{2} w_{1}\right)}{\left(r^{2}-a_{1}^{2}\right)^{2} \mathrm{P}_{1}^{2} \mathrm{P}_{2}^{2} \tan \theta_{0}}, \quad .
$$

and

$$
\mathrm{H}^{2}=\frac{4}{3} \frac{\pi^{2}\left(a^{2}+\frac{3}{32} d^{2}\right)\left(\mathrm{P}_{1}^{2} w_{2}+\mathrm{P}_{2}^{2} w_{1}\right)}{\left(r_{1}^{2}+a_{1}^{2}\right)^{\frac{3}{2}} \mathrm{P}_{1}^{2} \mathrm{P}_{2}^{2} \tan \theta_{1}}, . .
$$

where $P_{1}$ and $P_{2}$ are the periods of oscillation of the two deflectors respectively, $2 a_{1}$ the mean of their effective lengths (supposed nearly equal), and $w_{1}$ and $w_{2}$ their masses in grammes. Equations (9) to (12) give, of course, the same value for $\mathrm{H}$ if $a_{1}$ has been properly calculated. The value of $\frac{M}{w}$ is always calculated, as it gives an idea of the quality of steel used for the deflector. The results are given along with the values of $H$ in tabular form below.

The values of $\mathrm{H}$ derived from equations (9) to (12) require correction, in the oscillation experiment, for arc of vibration, for virtual increase of inertia due to air moved, and for change of inertia, due to change of dimensions of the deflector, if the temperatures when the bar is measured and when it is vibrated are different. Neither of these produced a sensible error in the experiments, and hence they were neglected. There are besides a correction for the effect of variation of temperature during the experiment in altering the magnetic moment of the deflector, and a correction for the change of magnetic moment between the oscillation and deflection experiment, due to the deflector having its length in the direction of the magnetic meridian in the former, and at right angles to it in the latter experiment. 
$492 \mathrm{Mr}$. T. Gray on Measurements of the Intensity of the

The magnets were found to change by $\frac{1}{21000}$ of their value per degree centigrade change of temperature; but as the change of temperature never exceeded two or three degrees, this correction was neglected.

The correction for induction, however, was found to be considerable in some cases, and the results tabulated below have all been corrected for that.

The Temperature Corffictent was obtained by placing the deflector behind the magnetometer-needle in such a position as to produce a deflection of 1000 scale-divisions, and the change of deflection produced by raising the temperature about $40^{\circ} \mathrm{C}$. observed.

The Induction Cokfficient was determined by placing the deflector, surrounded by a magnetizing coil, near the magnetometer-needle, and observing the change of deflection produced by passing a known current through the coil. The arrangement for these measurements is illustrated in Pl. XI. fig. 3 ; and the results of a number of experiments on the effect of the length and hardness of the deflector on this correction are given in Table II., and are shown diagrammatically in the following curve.

Curve illustrating the effect of Ratio of Length to Diameter on the Inductive Coefficient.

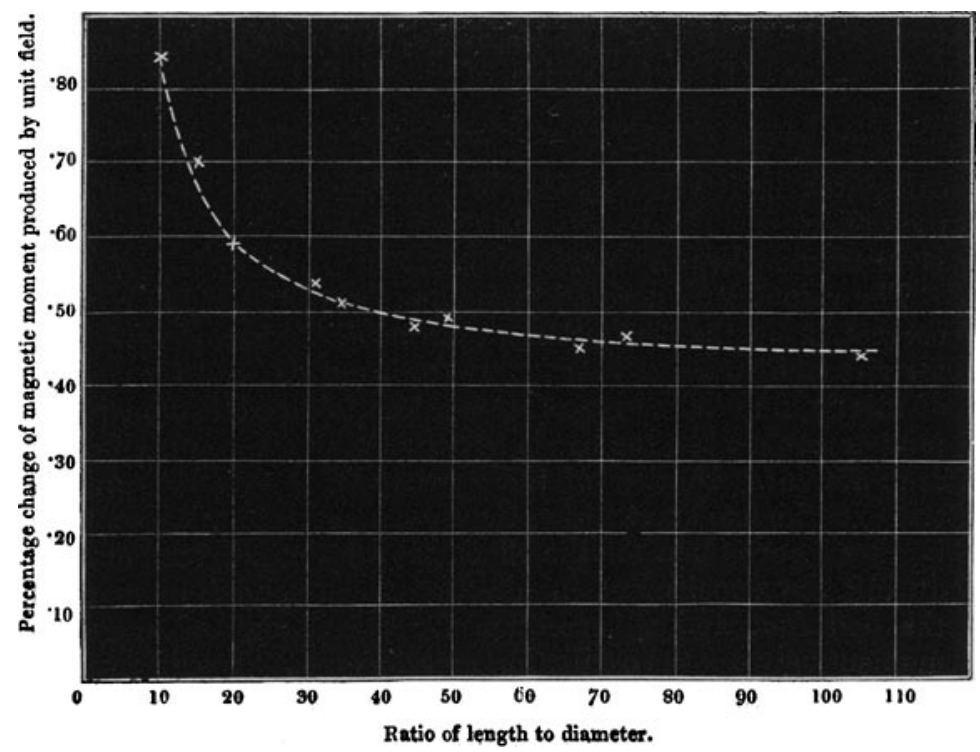


Referring to the figure, $m$ is the magnetometer-needle; $\mathrm{C}_{0}$ and $\mathrm{C}_{1}$ two coils, consisting of one layer of No. 30 B.W.G. silk-covered eopper wire, wound in glass tubes about 5 millim. in external diameter; $\mathrm{C}$ is a scale divided to millimetres, on which the deflection of the magnetometer-needle is read, in the manner described for the deflection-experiment above; $R$ is a box of resistance-coils, and $G$ a current-galvanometer. The line $\mathrm{DE}$ represents a magnetic meridian, and $\mathrm{AF}$ a line passing through the centre of $m$ and at right angles to $\mathrm{DE}$.

The coil $\mathrm{C}_{0}$ was placed with its axis parallel to $\mathrm{A} \mathrm{F}$, and its centre on the line $\mathrm{DE}$. The coil $\mathrm{C}_{1}$ was placed with its axis on the line $A F$, and at such a distance from $m$ that a current passed in the proper direclions through both coils produced no deflection. In making this adjustment a current, equal to about 30 times the greatest current subsequently used in the experiment, was passed through the coils.

The magnet to be tested was then introduced into one of the coils, adjusted to the proper position, and the deflection of $m$ read. A current of such strength as to produce a field of about $\frac{1}{10}$ C.G.S. units intensity was then passed through the coils, and the change of deflection read. The current was then reversed, and the deflection again read. This operation was repeated with stronger and stronger currents until a field of from one to two units intensity was reached. The magnet was next introduced into the other coil, and a similar series of measurements taken. The results plotted on a sheet of section-paper show clearly that a field of considerably greater intensity than that used is required to permanently alter the magnetic moment of the magnets when they are hardtempered.

The changes of deflection obtained when the magnet is in the coil $\mathrm{C}_{0}$ are always a smaller fraction of the total deflection than they are when it is in the coil $\mathrm{C}_{1}$. This is no doubt due to a change of magnetic distribution. The equations to the deflections in the two cases are :-

for the coil $\mathrm{C}_{0}$, and

$$
\theta=\frac{M}{H\left(r^{2}+a_{1}^{2}\right)^{\frac{3}{2}}}
$$

for the coil $\mathrm{C}_{1}$.

$$
\theta_{1}=\frac{2 r \mathrm{M}}{\mathrm{H}\left(r^{2}-a_{1}^{2}\right)^{2}}
$$

From these equations it is clear that either a change of $M$ or of $a_{1}$ will affect the deflection, but that the effect of the change of $a_{1}$ is opposite in the two cases. The method of measurement 


\section{$494 \mathrm{Mr}$. T. Gray on Measurements of the Intensity of the}

therefore allows both the change of moment $M$ and the change of effective length $2 a_{1}$ to be determined. When the change of moment only is required, this method has still an advantage in the fact that the effect of want of exact compensation between the two coils can be eliminated by means of the two measurements.

Sufficient sensibility is, for most purposes, obtained by using a deflection within the limits of the scale, but almost any degree of sensibility may be obtained by using an inferred zero. It is not advisable to place the magnets very near to $m$, because, if the tangent method be adopted, the compensation of the coils will not then be perfect unless $m$ is always brought to its original zero position; and if this objection be got over by using a sine method, in which the whole apparatus, including the magnetometer, can be turned round a vertical axis, there still remains the objection that the effect of distribution becomes very pronounced. An inferred zero method therefore involves, when small magnets are being tested, a diminution, by artificial means, of the intensity of the field at $m$. This, however, cannot be pushed far, on account of the very inconvenient fluctuations of zero which then take place, due to changes of declination in the earth's field, combined with the small variations of intensity of " $\mathrm{H}$ " being superposed on a field, the whole strength of which is not large compared with these variations.

The results of these experiments, given in Table II., show that it is of great importance, so far as induction is concerned, that the length of the magnets should be at least forty times their diameter, and that they should be made as hard as possible. It appears that for the steel employed in the experiments, which was of the kind commonly called silver steel, a much stronger magnet is obtained with a blue than with a glass-hard temper*. The magnets were originally magnetized by placing them between the poles of a large Ruhmkorff magnet excited by 24 tray-cells. They were again magnetized by placing them between the poles of the same magnet

* Note added October 26, 1885.- Since this paper was in type I have received a copy of No. 14 of the 'Bulletins' of the United States Geological Survey, which is wholly devoted to a discussion of experiments on the "Physical Characteristics of the Iron-Carburets," by C. Barus and V. Strouhal. In this work a large number of interesting experiments on the effect of annealing specimens of this "silver steel," which had been previously tempered glass-hard, are quoted. Among other things the large increase of magnetic moment which is obtained by softening the steal, up to a certain point, is clearly brought out.-T. G. 
excited by a dynamo having a low-resistance armature, and giving a potential of 100 volts. The relative strengths of the different magnets remained the same, and the strength only very slightly increased, thus showing that they were nearly magnetized to saturation.

The greatest trouble in the determination of the intensity of the earth's magnetic field arises out of the variations of the field itself-variations in declination causing changes of zero, and variations of intensity between different determinations causing apparent inaccuracy in the results.

This trouble was in the later measurements got over by means of a permanent magnetic vibrator, the period of vibration of which, at different times, gives a comparison of the intensity of the magnetic field at these times. In order to render the result of any one determination free from error due to diurnal variation, the period of the vibrator is taken at the beginning of the experiment, betwoen the deflection and the oscillation experiment, and again at the end of the experiment. If these agree well and the results also agree well among themselves, the value found is considered reliable; if not, the whole experiment is discarded. This vibrator has only lately beer adopted, and hence the results up to those taken on June 11th are not corrected for diurnal variation. The results are tabulated in the order in which they.were taken on each day; and it will be observed that the earlier results are generally the smaller, this being due to diurnal variation. The last three results given in the Table are corrected to noon for diurnal variation.

It has been found impossible to get consistent results at all on several occasions, owing to extraordinary variations, and this was notably the case on the 1st of September. It is interesting to note how readily the vibrator shows extraordinary variations which take place suddenly. The observer has only to watch for sudden changes of amplitude. These changes of amplitude are sometimes extremely marked, the vibrator being almost stopped on some occasions, and afterwards gradually worked up to a large swing.

Table I. gives the results of the various determinations. The meanings of the numbers in the different columns are clearly indicated in the headings. 
496 Horizontal Component of the Earth's Magnetic Field.

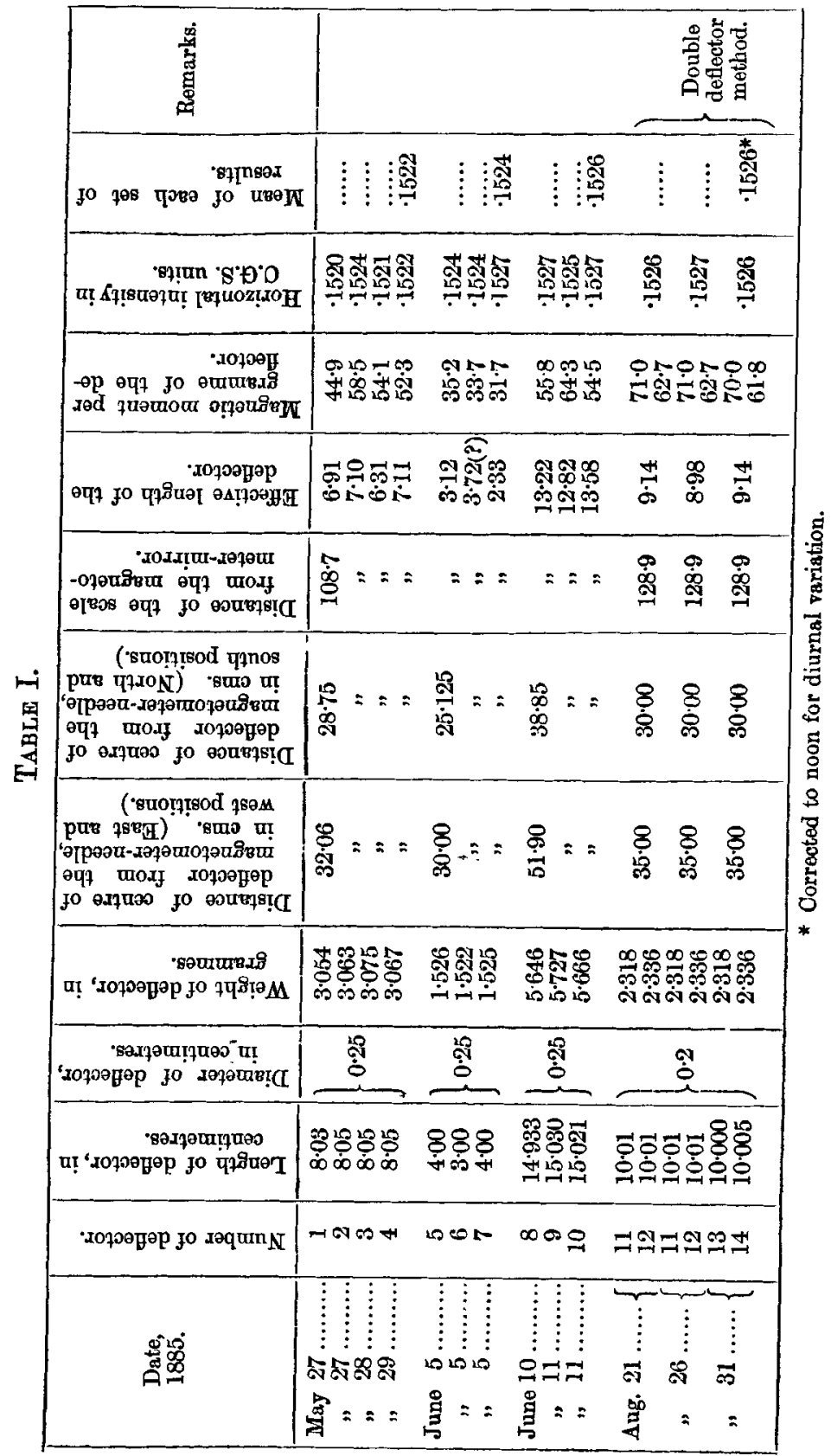


TABLE II.-Showing the effect of Length and of Hardness on the Induction-Coefficient of Magnets.

\begin{tabular}{|c|c|c|c|c|c|c|}
\hline \multirow[b]{2}{*}{$\begin{array}{l}\text { Length } \\
\text { of bar in } \\
\text { centi- } \\
\text { metres. }\end{array}$} & \multirow[b]{2}{*}{$\begin{array}{l}\text { Ratio of } \\
\text { length to } \\
\text { diameter. }\end{array}$} & \multicolumn{2}{|c|}{ Unit field. } & \multirow[b]{2}{*}{$\begin{array}{c}\text { Mean of } \\
\text { numbers in } \\
\text { columns } \\
3 \text { and } 4 .\end{array}$} & \multirow[b]{2}{*}{$\begin{array}{c}\text { Magnetic } \\
\text { moment } \\
\text { per } \\
\text { gramme. }\end{array}$} & \multirow[b]{2}{*}{ Remarks. } \\
\hline & & $\begin{array}{c}\text { Apparent } \\
\text { percentage } \\
\text { increase of } \\
\text { moment for } \\
\text { unit field: } \\
\text { side-on } \\
\text { position. }\end{array}$ & $\begin{array}{c}\text { Apparent } \\
\text { percentage } \\
\text { increase of } \\
\text { moment for } \\
\text { unit field: } \\
\text { end-on } \\
\text { position. }\end{array}$ & & & \\
\hline 3 & 10 & 0.80 & 0.90 & $0 \cdot 85$ & 27 & Glass hard \\
\hline 4 & 16 & $0 \cdot 67$ & 0.73 & 0.70 & 32 & $"$ \\
\hline 4 & 16 & 0.67 & $0 \cdot 70$ & $0 \cdot 69$ & 35 & $"$ \\
\hline 6 & 20 & 0.51 & 067 & $0 \cdot 59$ & 36 & $"$ \\
\hline 7 & 31 & 0.51 & 0.58 & 0.54 & 39 & " \\
\hline 8 & 32 & 0.51 & 0.58 & $0 \cdot 54$ & 54 & $"$ \\
\hline 8 & 32 & 0.51 & 0.58 & 0.54 & 52 & ", \\
\hline 10 & 34 & $9 \cdot 46$ & 0.56 & 0.51 & 40 & " \\
\hline 10 & 44 & 0.40 & $0 \cdot 56$ & 0.48 & 43 & $"$ \\
\hline 7 & 47 & $0 \cdot 46$ & $0 \cdot 51$ & $0 \cdot 49$ & 57 & $"$ \\
\hline 10 & 50 & 0.44 & 0.58 & 0.51 & 67 & $"$ \\
\hline 10 & 50 & 0.48 & 0.54 & 0.51 & 60 & $"$ \\
\hline 10 & 50 & $0 \cdot 46$ & 0.55 & 0.51 & 53 & $"$ \\
\hline 10 & 50 & 0.46 & 0.52 & 0.49 & 71 & , \\
\hline 10 & 50 & $0 \cdot 46$ & 0.56 & 0.51 & 60 & $"$ \\
\hline 10 & 67 & 0.41 & 0.51 & $0 \cdot 46$ & 65 & $"$ \\
\hline 7 & 73 & $0 \cdot 41$ & 0.50 & 0.47 & 64 & $"$ \\
\hline 10 & 105 & 0.42 & 0.45 & 0.43 & 66 & ", \\
\hline 10 & 34 & $0 \cdot 47$ & 0.53 & 0.50 & $41 \cdot 5$ & Glass hard. \\
\hline 10 & 34 & $0 \cdot 63$ & 0.67 & $0 \cdot 65$ & $44 \cdot 5$ & Yellow. \\
\hline 10 & 34 & 0.84 & 0.98 & 0.91 & $54 \cdot 1$ & Blue. \\
\hline 10 & 48 & $0 \cdot 32$ & 0.40 & $0 \cdot 36$ & 45 & Glass hard. \\
\hline 10 & 48 & 0.43 & 0.55 & 0.49 & 46 & Yellow. \\
\hline 10 & 48 & 0.53 & 0.67 & 0.60 & 71 & \\
\hline
\end{tabular}

LX. The Periodic Law, as Illustrated by certain Physical Properties of Organic Compounds.-Part II. The Meltingand Boiling-points of the Halogen and Alkyl Compounds of the Hydrocarbon Radicals. By Thomas CaRneliey, D.Sc., Professor of Chemistry in University College, Dundee*.

TN the present series of papers my object is to determine 1 whether the elements are in any way analogous to the hydrocarbon radicals of Organic Chemistry. For this purpose

* Communicated by the Author. 